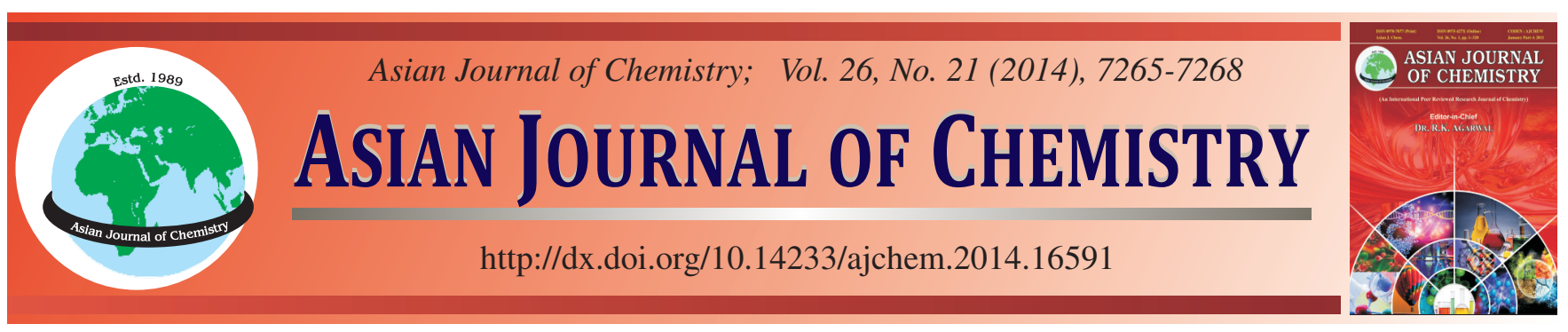

\title{
Effect of Membrane Structure on Blood Compatibility for Polyethersulfone Hollow Fiber Membrane
}

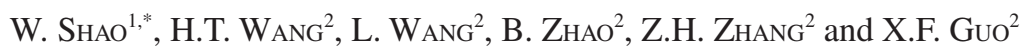

${ }^{1}$ Tianjin Municipal Key Lab of Fiber Modification and Functional Fiber, Institute of Functional Fibers, Tianjin Polytechnic University, Tianjin 300387, China

${ }^{2}$ School of Environmental and Chemical Engineering, Tianjin Polytechnic University, Tianjin 300387, P.R. China

*Corresponding author: E-mail: 1xb3779@126.com

Received: 28 October 2013;

Accepted: 11 January 2014;

Published online: 30 September 2014;

AJC-16116

\begin{abstract}
In this study, polyethersulfone membranes with four types of structures, which were double finger structure (PES-DF), single finger structure (PES-SF), compact sponginess structure (PES-CS) and loose sponginess structure (PES-LS), were obtained by changing the additives and spinning techniques. Effect of the polyethersulfone membranes' structure on its blood compatibility was investigated by the experiments of water contact angle, water adsorption, bovine serum albumin adsorption and blood clotting time. All results showed that polyethersulfone membranes with sponginess structure had better blood compatibility than polyethersulfone membranes with finger structure. As for the polyethersulfone membranes with sponginess structure had better blood compatibility than double finger structure.
\end{abstract}

Keywords: Membrane structure, Polyethersulfone, Blood compatibility.

\section{INTRODUCTION}

Hemodialysis is one of the most important methods for uraemia and renal failure. Hemodialysis membrane is the hardcore for dialysis. The membrane material for hemodialysis needs having good blood compatibility besides thermal stability, mechanical strength, chemical inertness and membraneformation feasibility due to the contact between the membrane material and body blood in the process of dialysis. At present, as for the poor blood compatibility of dialysis membranes, many efforts have been made to modify the membranes by introducing other groups in basic membrane material through chemical reaction $^{1-4}$. The polyvinylpyrrolidone (PVP), polyethylene glycol (PEG), human blood albumin, chitosan, phospholipid and some substance possessing anticoagulant properties were commonly used as macromolecule material in the material modification. All the efforts improved the hydrophilicity and blood compatibility of hemodialysis membrane to a certain extent and promoted the development of hollow fiber hemodialysis membranes.

Recently, our research group found that double finger structure (PES-DF), single finger structure (PES-SF), compact sponginess structure (PES-CS) and loose sponginess structure (PES-LS) may be obtained by changing the additives and spinning techniques during the research of surface modification for polyethersulfone hollow fiber hemodialysis membranes ${ }^{5}$. In the clinical application of hemodialysis membrane, the membrane and human blood contact directly. Useful substance in the human blood such as human blood albumin and blood platelet were recovered, but water, inorganic salt, toxin and low-molecular weight proteins such as $\beta_{2}$-microglobulin (MW $11800 \mathrm{Da})$ passed through the membrane and removed with the dialysate. Due to the direct contact between the inner of membrane hole and blood in the whole process of dialysis, close connection exists between the membrane structure and blood compatibility. However, related researches about the effect of membrane structure on blood compatibility have not been reported so far.

In this study, polyethersulfone hollow fiber hemodialysis membranes with four different structures were prepared by changing the additive and spinning techniques. The experiments of water contact angle, bovine serum albumin adsorption and blood clotting time were conducted to investigate contrastively in order to discuss the relation between the membrane structure and its blood compatibility.

\begin{tabular}{l}
\hline EXPERIMENTAL \\
\hline Polyethersulfone (RADEL ${ }^{\circledR} \mathrm{A}, \mathrm{MW}=58,000$, flake) was \\
purchased from Solvay Co. Polyethylene glycols (PEG-400, \\
PEG-20000) were purchased from Tianjin GUANG FU \\
Institute of Fine Chemicals. $N$-Methyl-2-pyrrolidone (NMP) \\
(Samsung Fine Chemicals) was used as solvent. Bovine serum \\
albumin (BSA, $67 \mathrm{kDa}$ ) was obtained from Institute of Blood \\
for Chinese Academy of Medical Science Co., Ltd. Sodium
\end{tabular}


dodecyl sulfate (SDS) was supplied by Tianjin Kemiou Co., Ltd. Bicinchoninic acid (BCA) was purchased from Beijing Biosynthesis Biotechnology Co., Ltd.

Preparation of the hollow fiber hemodialysis membranes with different structures: Polyethersulfone flakes and macromolecular hydrophilic additives were dried for $20 \mathrm{~h}$ at $105^{\circ} \mathrm{C}$ in vacuum oven and then moved into the four mouth flasks. PEGs as pore forming agents and $\mathrm{N}$-methyl pyrrolidone (NMP) as solvent were added to the flasks, in which PEG-400 was used for preparing the polyethersulfone membrane with finger structure and PEG-20000 for the polyethersulfone membrane with sponginess structure. Then the spinning solution was stirred for $24 \mathrm{~h}$ at $70{ }^{\circ} \mathrm{C}$ and allowed to stand overnight to remove air bubbles. After the spinning solution was moved into the spinning equipment, the polyethersulfone membranes with different structures were prepared by controlling the spinning tank pressure, core flow rate, coagulation bath temperature, air gap distance, the temperature of filter and spinneret and the winding speed. In addition to PEG-400, PES-DF and PES-SF were obtained by controlling above parameters. For another additive PEG-20000, PES-CS and PES-LS can obtained accordingly. Different structures of polyethersulfone hollow fiber membranes were placed properly for the process of analysis and detection after the experiment.

As the inner diameter and outer diameter of polyethersulfone hollow fiber hemodialysis membranes prepared in this experiment were 200 and $300 \mu \mathrm{m}$ respectively, the water contact angle can't measured. So plate membrane prepared by the casting membrane solution instead of the spinning solution, having the same mixing ratio as hollow fiber membrane, was used in contact angle measurement. The preparation method for plate membrane has explained already in our previous research ${ }^{6}$.

Membranes characterization: The cross-section morphologies of membranes with different structure were observed by scanning electron microscopy (SEM) using a XL 30ESME scanning microscope. The membranes frozen in liquid nitrogen were broken and sputtered with gold before SEM analysis.

Hydrophilicity measurements: The surface hydrophilicity of the polyethersulfone membranes with different structures were characterized on the basis of contact angle and water absorption measurements. Using a sessile drop method, water contact angle was measured at room temperature by a contact angle goniometer (JY-82). $15 \mu \mathrm{L}$ deionized water was dropped onto a dry membrane using a microsyringe at atmosphere of saturated water vapor and then contact angle was measured after $10 \mathrm{~s}$. At least 5 contact angles were averaged to get a reliable value. Water absorption was defined as w1 and $\mathrm{w} 2$, where $\mathrm{w} 1$ and $\mathrm{w} 2$ represent the weight of the dry membrane $(1 \mathrm{~cm} \times 1 \mathrm{~cm})$ and the membrane was soaked in deionized water for $12 \mathrm{~h}$ at $25 \pm 0.5^{\circ} \mathrm{C}$, respectively. The reported value was the average of at least 5 experiments.

Bovine serum albumin adsorption assay on the membrane: The polyethersulfone membranes with different structure (outer diameter was $300 \mu \mathrm{m}$, the length was $10.62 \mathrm{~cm}$ and the membrane area was $1 \mathrm{~cm}^{2}$ ) were immersed in phosphate buffer solution $(0.02 \mathrm{M}, \mathrm{pH}=7.4)$ of $5000 \mathrm{ppm}$ bovine serum albumin for $4 \mathrm{~h}$ at $37^{\circ} \mathrm{C}$. Then the membranes were rinsed three times with phosphate buffer solution and inserted into a glass tube containing $1 \mathrm{wt} \%$ aqueous solution of SDS. The glass tubes containing the membranes and SDS solution were shaken for $2 \mathrm{~h}$ at room temperature to remove the proteins adsorbed on the membranes. The amount of proteins adsorbed on the surface of membranes was calculated from the concentration of proteins in the SDS solution using a protein analysis kit.

Blood coagulation time: The in vitro coagulation times, including activated partial thrombin time (APTT) and prothrombin time (PT), were determined using an automated blood coagulation analyzer (CA-50, Sysmex Corp., Kobe, Japan). Blood coagulation times were measured as follows: the sample membrane (outer diameter was $300 \mu \mathrm{m}$, the length was 10.62 $\mathrm{cm}$ and the membrane area was $1 \mathrm{~cm}^{2}$ ) was placed carefully in tubes and incubated in $0.5 \mathrm{~mL}$ of platelet-poor plasma (PPP) at $37{ }^{\circ} \mathrm{C}$ for $0.5 \mathrm{~h}$. Afterward, $0.1 \mathrm{~mL}$ residual PPP solution was mixed well with $0.1 \mathrm{~mL}$ of Dade Actin ${ }^{\circledR} \mathrm{FS}$ (Date Behring Marburg $\mathrm{GmbH}$ ) agent at $37^{\circ} \mathrm{C}$ for $3 \mathrm{~min} . \mathrm{CaCl}_{2}$ was added into the tube and the solution was mixed well. After $20 \mathrm{~s}$, the time of clot formation was recorded with a chronometer. The tests were repeated three times for each sample.

\section{RESULTS AND DISCUSSION}

Preparation of hollow fiber hemodialysis membrane with different structures: Polyethersulfone was used as membrane material and polyethersulfone hollow fiber hemodialysis membranes with four different structures were obtained by changing the additives and spinning techniques. Fig. 1a shows the pictures of polyethersulfone membrane with double finger structure. Fig. 1b shows the pictures of polyethersulfone membrane with single finger structure. Fig. 1c shows the pictures of polyethersulfone membrane with compact sponginess structure. Fig. 1d shows the pictures of polyethersulfone membrane with loose sponginess structure. In order to distinguish conveniently, 4 kinds of structures for polyethersulfone membrane were named PES-DF, PES-SF, PES-CS and PESLS in turn.

Filtration and rejection properties: Table- 1 shows the results of water flux, bovine serum albumin solution flux and bovine serum albumin rejection in order to investigate the filtration performance of polyethersulfone hemodialysis membranes with different structures. It was found that the bovine serum albumin rejection of all polyethersulfone membranes was more than $99.5 \%$. So there was a reason to believe that all four kinds of polyethersulfone membranes can reject bovine serum albumin absolutely. Diffferent additives and spinning techniques were used in order to obtain four kinds of polyethersulfone hemodialysis membrane in the preparation process of membranes. So the data of pure water flux and bovine serum albumin solution flux lacked comparability and were not analyzed herein.

Hydrophilicity of membranes: Water contact angle has been commonly used to evaluate the relative hydrophilicity or hydrophobicity of the membrane surface. Low contact angle value normally means high hydrophilicity. Fig. 2 shows the result of water contact angle for polyethersulfone membranes with different structures. It can be seen that the water contact angle of PES-DF, PES-SF, PES-CS and PES-LS was 57, 59 ${ }^{\circ}$, $42^{\circ}$ and $45^{\circ}$ respectively. Membranes with sponginess structure have good hydrophilicity than the membrans with finger 

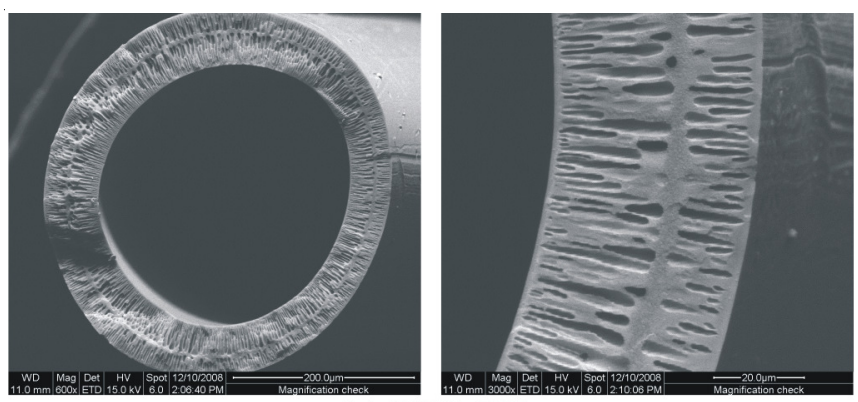

(a) PES-DF
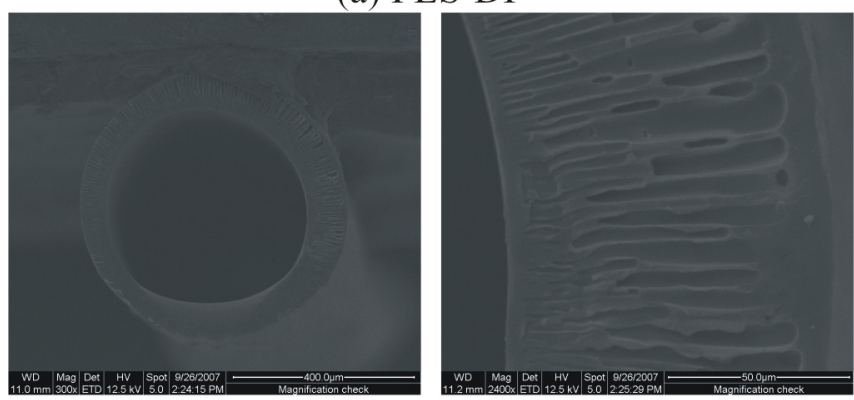

(b) PES-SF
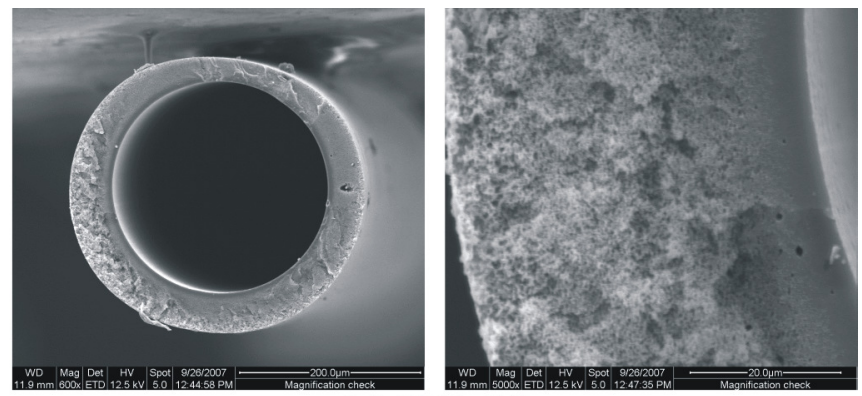

(c) PES-CS
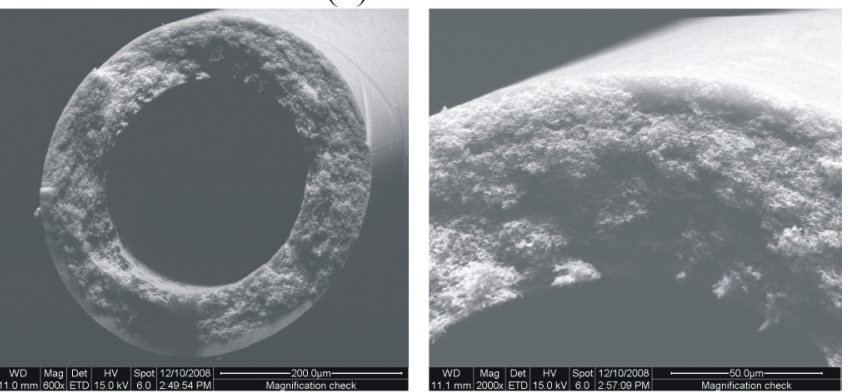

(d) PES-LS

Fig. 1. Pictures of polyethersulfone membrane structure; (a) PES-DF; (b) PES-SF; (c) PES-CS; (d) PES-LS

TABLE-1

FILTRATION PERFORMANCES OF THE POLYETHERSULFONE MEMBRANES WITH DIFFERENT STRUCTURE ${ }^{a}$

\begin{tabular}{cccc}
\hline Membrane & $\mathrm{J}_{\mathrm{w}}\left(\mathrm{L} / \mathrm{m}^{2} \mathrm{~h}\right)^{\mathrm{b}}$ & $\mathrm{J}_{\mathrm{b}}\left(\mathrm{L} / \mathrm{m}^{2} \mathrm{~h}\right)^{\mathrm{b}}$ & $\begin{array}{c}\text { Retention of bovine } \\
\text { serum albumin }(\%)\end{array}$ \\
\hline PES-DF & $58.9 \pm 2.5$ & $21.2 \pm 1.2$ & 99.9 \\
PES-SF & $84.2 \pm 3.5$ & $35.5 \pm 1.8$ & 99.8 \\
PES-CS & $120.6 \pm 4.7$ & $52.8 \pm 2.6$ & 99.9 \\
PES-LS & $188.5 \pm 5.5$ & $76.5 \pm 3.5$ & 99.9 \\
\hline
\end{tabular}

${ }^{a}$ The filtration test was conducted at a constant pressure of $0.1 \mathrm{MPa}$ and a system temperature of $37 \pm 0.5^{\circ} \mathrm{C},{ }^{b} \mathrm{~J}_{\mathrm{w}}$ : water flux; $\mathrm{J}_{\mathrm{b}}$ : bovine serum albumin solution flux

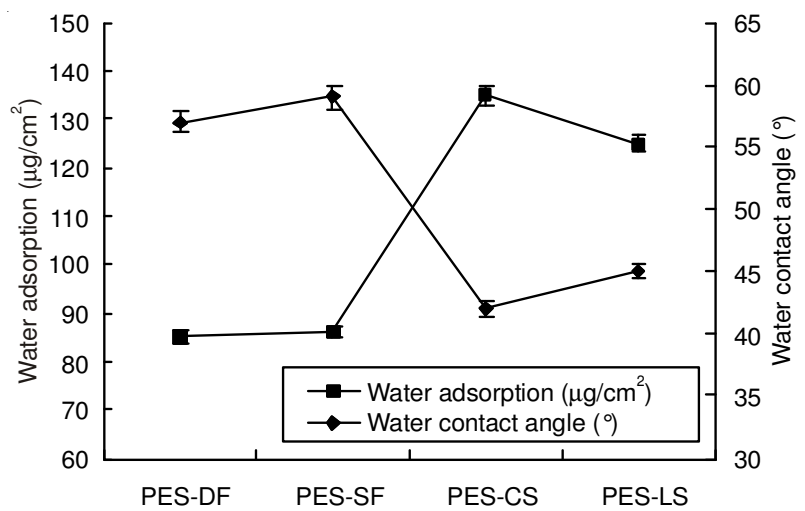

Fig. 2. Water contact angle and water absorption for polyethersulfone membranes with different structure

structure. As for the membranes with sponginess structure, PES-CS had good hydrophilicity than PES-LS.

Membrane water absorption is another important factor to characterize the relative hydrophilicity or hydrophobicity of the membrane surface ${ }^{7}$. Higher water adsorption per unit area commonly means better hydrophilicity. On the contrary, lower water adsorption per unit area means poor hydrophilicity. It can be seen form Fig. 2 that the water adsorption of membranes with sponginess structure obviously higher than that of membrans with finger structure. As the same membranes with sponginess structure, PES-CS had higher water adsorption than PES-LS. Loose sponginess structure had the best hydrophilicity in all the four membranes. This result was consistent with that of water contact angle above.

Bovine serum albumin adsorption on polyethersulfone membranes: Protein adsorption on the materials is a key phenomenon during thrombogenic formation. Materials having good blood compatibility commonly can inhibit the protein adsorption when they contact with body blood. In this way, blood coagulation and complement actication caused by materails happened rarely. So the amount of proteins adsorbed on the membrane materials is one of the important factors in evaluating the blood compatibility of materials.

Fig. 3 shows the adsorption of bovine serum albumin on polyethersulfone membranes with different structures. It was found that membranes with sponginess structure have lower bovine serum albumin adsorption than the membranes with finger structure. PES-CS had the lowest bovine serum albumin adsorption. Above results indicate that polyethersulfone

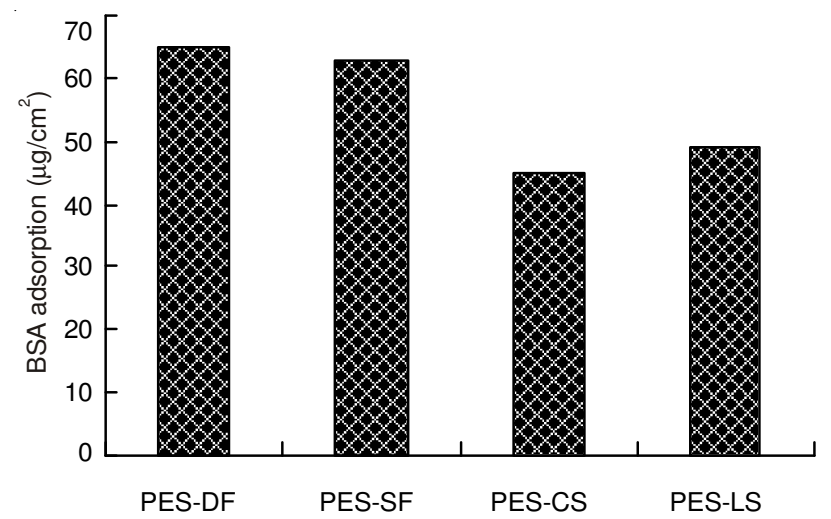

Fig. 3. Adsorption of bovine serum albumin on polyethersulfone membranes with different structure 
membranes with sponginess structure inhibited more protein on the membrane surface compared with membranes with finger structure. Because of this, membranes with sponginess structure had better blood compatibility than the membranes with finger structure.

Anti-coagulant properties: Clotting time is another factor to investigate the blood compatibility of the membrane materials. Blood coagulation unavoidably happened when the material surface directly contact the blood. Longer blood clotting time means that membrane material owns good blood compatibility. Fig. 4 shows the results of the clotting times for polyethersulfone membranes with four different structure. It can be seen that the clotting times of PES-CS, PES-LS was 86 $\mathrm{s}$ and $83 \mathrm{~s}$ respectively, much longer than that of the PES-DF and PES-SF. The above results also show that polyethersulfone membranes with sponginess structure have better blood compatibility than that of polyethersulfone membranes with finger structure.

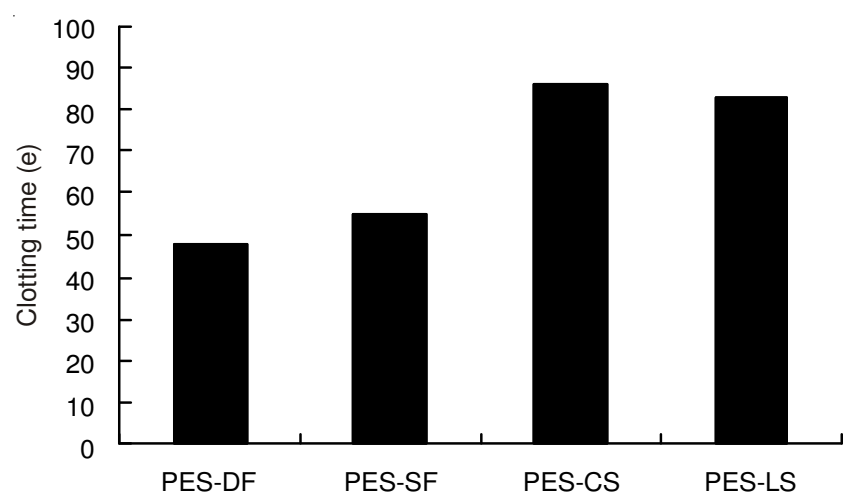

Fig. 4. Comparison of blood clotting time of polyethersulfone membranes with different structure

\section{Conclusion}

In this study, polyethersulfone membrane with double finger structure (PES-DF), polyethersulfone membrane with single finger structure (PES-SF), polyethersulfone membrane with compact sponginess structure (PES-CS) and polyethersulfone membrane with loose sponginess structure (PES-LS) were obtained by changing the additive and spinning techniques. Based on this, effect of the membrane structure on blood compatibility for polyethersulfone membrane was investigated. All results show that polyethersulfone membranes with sponginess structure have better blood compatibility than that of polyethersulfone membranes with finger structure by the experiments of water contact angle, water adsorption, bovine serum albumin adsorption and blood clotting time. As for the polyethersulfone membranes with sponginess structure, PESCS had better blood compatibility than that of PES-LS. However, polyethersulfone membranes with sponginess structure have better blood compatibility than that of polyethersulfone membranes with finger structure. The influence mechanism of the related research will be developed in the future by our research group.

\section{ACKNOWLEDGEMENTS}

This work was financially supported by the National Natural Science Fund of China $(51138008,51108315,21206125)$ and the Tianjin Technology Support Program (11ZCGYSF01500).

\section{REFERENCES}

1. B.H. Fang, Q.Y. Ling, W.F. Zhao, Y. Ma, P. Bai, Q. Wei, H. Li and C. Zhao, J. Membr. Sci., 329, 46 (2009).

2. F. Ran, S.Q. Nie, W.F. Zhao, J. Li, B. Su, S. Sun and C. Zhao, Acta Biomater., 7, 3370 (2011).

3. W.C. Lin, D.G. Yu and M.C. Yang, Colloids Surf. B, 44, 82 (2005).

4. D.P. Lu, H. Zou, R. Guan, H. Dai and L. Lu, Polym. Bull., 54, 21 (2005).

5. H.T. Wang, Doctoral Dissertation, Tianjin Polytechnic University, Tianjin, China (2010).

6. L.Z. Zhang, L.F. Yang and H.T. Wang, Polym. Mater. Sci. Eng., 26, 40 (2010).

7. Z.K. Xu, F.Q. Nie, C. Qu, L.-S. Wan, J. Wu and K. Yao, Biomaterials, 26, 589 (2005). 